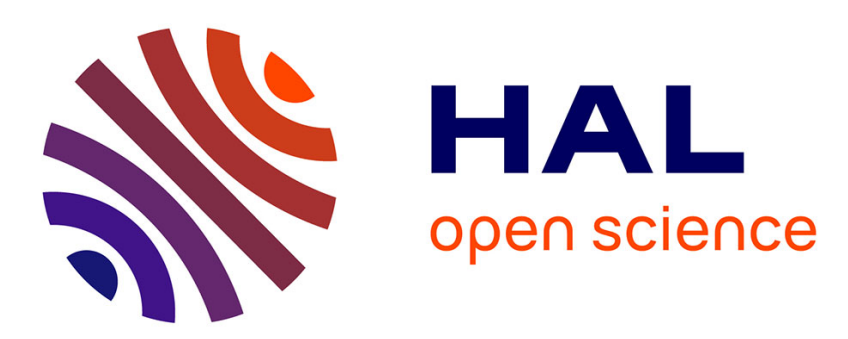

\title{
Magnon excitations and the specific heat of EuxSr1- xS spin glasses in strong magnetic fields \\ U. Krey
}

\section{To cite this version:}

U. Krey. Magnon excitations and the specific heat of EuxSr1- xS spin glasses in strong magnetic fields. Journal de Physique Lettres, 1985, 46 (17), pp.845-850. 10.1051/jphyslet:019850046017084500 . jpa00232908

\section{HAL Id: jpa-00232908 https://hal.science/jpa-00232908}

Submitted on 1 Jan 1985

HAL is a multi-disciplinary open access archive for the deposit and dissemination of scientific research documents, whether they are published or not. The documents may come from teaching and research institutions in France or abroad, or from public or private research centers.
L'archive ouverte pluridisciplinaire HAL, est destinée au dépôt et à la diffusion de documents scientifiques de niveau recherche, publiés ou non, émanant des établissements d'enseignement et de recherche français ou étrangers, des laboratoires publics ou privés. 


\title{
Magnon excitations and the specific heat of $\mathrm{Eu}_{x} \mathrm{Sr}_{1-x} \mathrm{~S}$ spin glasses in strong magnetic fields
}

\author{
U. Krey \\ Fakultät für Physik der Universität Regensburg, D-8400 Regensburg, F.R.G.
}

(Reçu le 20 mai 1985, accepté sous forme définitive le 10 juillet 1985)

\begin{abstract}
Résumé. - Des excitations collectives de spin semblables aux magnons sont étudiées pour les verres de spin $\mathrm{Eu}_{x} \mathrm{Sr}_{1-x} \mathrm{~S}$, avec $x=0,25,0,44$ et 0,54 , sous un champ magnétique très fort $(B>3 \mathrm{~T})$. On trouve que pour ces champs forts les excitations quasi-magnons peuvent être traitées comme des bosons non-interagissants bien au-dessus de la température de transition verre de spin à $B=0$. Nous calculons sans paramètre ajustable la densité d'état des magnons et la partie magnétique de la chaleur spécifique, obtenant un accord excellent avec des mesures récentes, entre $T=0,3$ et $5 \mathrm{~K}$ (10 K), pour $B=3,6 \mathrm{~T}(6,6 \mathrm{~T})$.

Abstract. - The density of states of magnon-like excitations of $\mathrm{Eu}_{x} \mathrm{Sr}_{1-x} \mathrm{~S}$ spin glasses with $x=0.25$, 0.44 and 0.54 is calculated for very strong magnetic fields $B$, i.e. $B>3 \mathrm{~T}$. It is found that for these high fields the magnon-like excitations can be treated as non-interacting bosons up to temperatures far beyond the spin glass freezing temperature at $B=0$. From the density of states the magnetic part $C_{\mathbf{H}}$ of the specific heat is calculated, without any fit-parameter. The results agree quantitatively with recent experiments, for $T$ between $0.3 \mathrm{~K}$ and $5 \mathrm{~K}(10 \mathrm{~K})$ in magnetic fields of $3.6 \mathrm{~T}(6.6 \mathrm{~T})$.
\end{abstract}

\section{Introduction.}

For several years already, collective spin excitations in the $\mathrm{Eu}_{x} \mathrm{Sr}_{1-x} \mathrm{~S}$ system have raised considerable interest [1]. The reason is that (i) the $\mathrm{Eu}_{x} \mathrm{Sr}_{1-x} \mathrm{~S}$ system, at $T=0$ and zero external field $B$, shows spin glass behaviour for $x$ between 0.13 and 0.65 , see [1], while (ii) in contrast to other spin glasses the interactions are short ranged and completly known. Therefore, the $\mathrm{Eu}_{x} \mathrm{Sr}_{1-x} \mathrm{~S}$ system is particularly suited for a quantitative comparison between experiment and theory.

In fact, in three papers [2-4], the present author has studied the collective excitations of the above-mentioned system numerically, by means of a continued fraction algorithm. The resulting density of states, in a noninteracting-boson approximation, leads to predictions for the specific heat, which were in quantitative agreement with measurements of Meschede et al. [5]. Similar theoretical results have been obtained in [6] by somewhat different methods.

However, these former calculations and the measurements were concentrated on the case of vanishing magnetic field $B$ : in that case the non-interacting Bose approximation yields sensible results only for temperatures $T \lesssim T_{\mathrm{f}}$, where $T_{\mathrm{f}}$ is the freezing temperature of the system (typically 1-2 K). Moreover, the calculation is rather complex, since one has to generate at first a ground state configuration of the spin glass, while the excited states had to be analysed afterwards 
in the RPA approximation, i.e. from the linearized equations of motion for the deviations from the complicated ground state.

Very recently, however, the measurements have been extended to very high external fields, up to $6 \mathrm{~T}$ [7], and additionally also the higher temperatures, up to $10 \mathrm{~K}$ [8]. The results are interesting in several respects, the most interesting feature being perhaps the evidence for a gap in the excitation spectrum, [7, 8]. Moreover, the gap energy seemed to be strongly related to the external field. However, while in reference [7] there was no quantitative theoretical interpretation, the interpretation in [8] is rather crude and unsatisfactory, both in qualitative and quantitative respect. The authors use a simple Schottky anomaly, i.e. an incoherent superposition of identical single spin excitations with excitation energy at $E=g \mu_{\mathrm{B}}\left(B+B_{0}\right)$, to fit the experiments, ending with a fit parameter of $B_{0}=3.4 \mathrm{~T}$, for which they try to give some qualitative reasoning.

In the present Letter, in contrast, it is shown that the experimental results of [7] and [8] can be explained, without any fit parameter, from a direct numerical calculation of the collective excitations of the system. In fact, for the high external fields of 3 and $6 \mathrm{~T}$ the ground state of the spin system is known and very simple (namely all spins are aligned), and the low-lying collective excitations are magnon-like (i.e. noninteracting bosons, see below !). Of course, since one is dealing with a strongly disordered system, these magnon-like excitations cannot be labelled by the quasi-momentum q.

\section{Theoretical definitions.}

The Hamiltonian characterizing the magnon eigenmodes is simply

$$
\mathbb{H}=\sum_{l, n} H_{l, n}|l\rangle\langle n| \text {. }
$$

Here $l$ and $n$ run over the Eu sites only, and $|l\rangle$ is the state where at site $l$ the magnetic quantum number $m$, characterizing the $z$-component of the spin, is reduced from the maximum value $m=s$ $(=7 / 2)$ to $m=s-1$. Furthermore, $H_{l, n}$ is as usual given by

$$
H_{l, n}=2 s\left(\sum_{p} J_{l, p} \delta_{l, n}-J_{l, n}\right)+g \mu_{\mathrm{B}} B \delta_{l, n},
$$

where the last term on the r.h.s. represents the Zeeman energy. The eigenmodes $|v\rangle$ of $\forall$ are obtained as usual by the ansatz $|v\rangle=\sum_{l} c_{l}|l\rangle$, i.e. by a coherent superposition of single-site states, and not by the incoherent single spin excitations invoked in [8].

Now, the exchange interactions $J_{l, n}$ appearing in (2) are known, namely $J_{l, n}=J_{1} \cong 0.22 \mathrm{~K}$, if $l$ and $n$ are nearest neighbours, and $=J_{2} \cong-0.11 \mathrm{~K}$, if $l$ and $n$ are second-nearest neighbours. Further ranging interactions can be neglected (see [4] !).

\section{Results : magnon density of states.}

I have generated three computer models of $\mathrm{Eu}_{x} \mathrm{Sr}_{1-x} \mathrm{~S}$ crystals with $x=0.25,0.44$, and 0.54 , respectively, by distributing $\mathrm{Eu}$ and $\mathrm{Sr}$ atom randomly on the sites of a fcc lattice; the total number of fcc sites was $20^{3}$, and periodic boundary conditions have been used to enhance the quality of results. Then, with the Hamiltonian (1) the normalized density of states $g(E)$ is calculated by means of the continued fraction method.

It is not necessary to describe the continued fraction method at this place, since it is well known, see e.g. [9]. It is particularly suited for excitations in disordered systems, and for spin glasses at $B=0$ it has already been used extensively by the present author, see [2-4]. In the present case, for the calculation of $g(E)$ the method of random starting states has been used (see [2-4]). The conti- 
nued fraction coefficients $a_{n}$ and $b_{n}$ have been calculated up to $n=8$, corresponding to 16 exact moments, while for larger $n, a_{n}$ and $b_{n}$ have been replaced as usual by $a_{8}$ and $b_{8}$, respectively.

Figure 1 shows the results for $g(E)$, for $x=0.25,0.44$, and 0.54 . The energy $E$ has been expressed in units of $\mathrm{K}$. Bor the internal magnetic field a value of $B=6.6 \mathrm{~T}$ has been used, corresponding to the measurements of 8 . Actually this is no restriction, since a change $\delta B$ would simply lead to a rigid shift of $g(E)$, i.e. the argument $E$ would be shifted by $\delta E=g \mu_{\mathrm{B}} \delta B$ with $g=2$, as long as in the ground state the spins remain practically aligned (i.e. as long as the gap appearing in the excitation spectrum remains large enough, see below !).

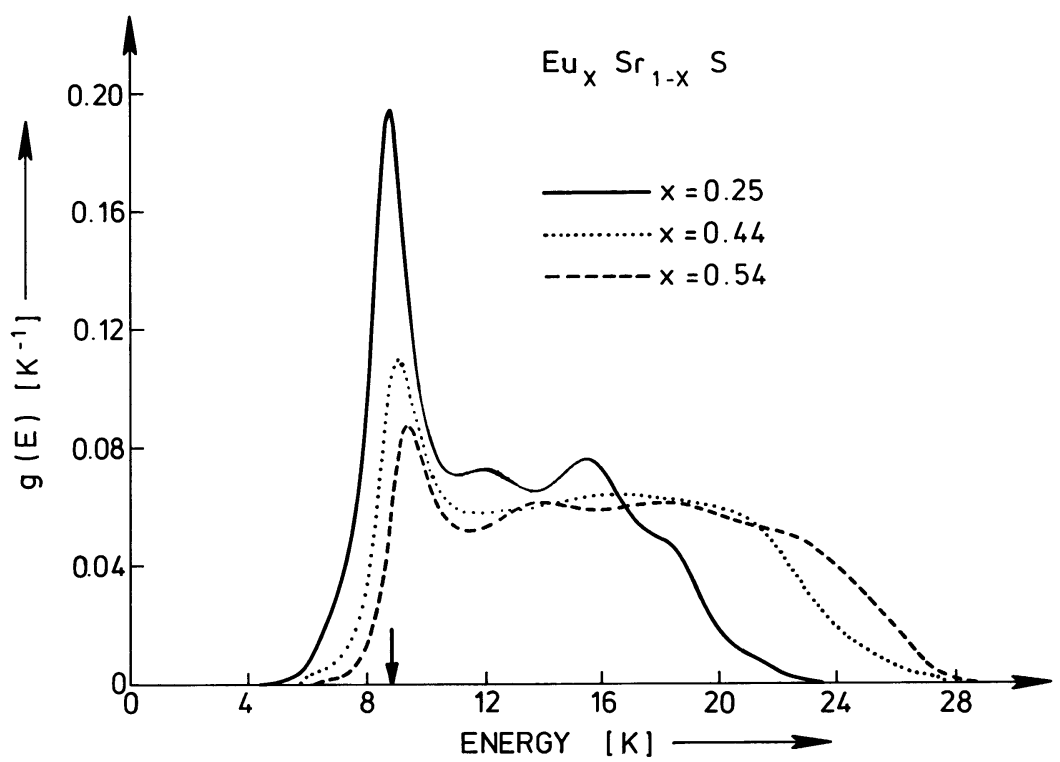

Fig. 1. - The normalized level-density $g(E)$ of magnon-like states, i.e. eigenstates of the Hamiltonian (1), is presented over the energy $E$ for $\mathrm{Eu}_{x} \mathrm{Sr}_{1-x} \mathrm{~S}$ crystals with $x=0.25,0.44$ and 0.54 . The energies have been expressed in units of K, i.e. $E$ means $E / k_{\mathrm{B}}$, with Boltzmann's constant $k_{\mathrm{B}}$. The calculation was performed for a magnetic field of $B=6.6 \mathrm{~T}$. The arrow at $E=8.87 \mathrm{~K}$ denotes the Zeeman energy corresponding to this field.

In fact, the first interesting observation in figure 1 is that in all three cases there is a gap in the spectrum. However, the gap energy $E_{\mathrm{g}}$ does not agree with the Zeeman energy $E_{B}:=g \mu_{\mathrm{B}} B$ (which would correspond to $8.87 \mathrm{~K}$ for $B=6.6 \mathrm{~T}$ ), but is considerably smaller, e.g. for $x=0.25$, 0.44 , and 0.54 it is as small as $4.4,5.75$, and $6.25 \mathrm{~K}$, respectively (see Fig. 1 !).

The reason for the smallness of $E_{\mathrm{g}}$ is simple : only if all exchange constants would be $\geqslant 0$, the gap energy would be equal to $E_{B}$, whereas for the present system, at $B=0$, the negative nearest neighbour exchange together with the disorder would lead to a spin glass ground state, i.e. the aligned state can remain the ground state only for sufficiently strong fields, i.e. as long as the gap remains open. For $x=0.25,0.44$, and 0.54 this should be the case for $B \gtrsim 3.3,2.3$, and $2 \mathrm{~T}$, respectively.

The second obvious feature in figure 1 is the strong peak of $g(E)$ around $E=E_{B}$, which becomes more and more pronounced for decreasing $x$. This peak corresponds to strongly "frustrated " spins, which would rotate almost freely, if the field $B$ would be switched off. Finally, in figure 1 , it is important to note that a very large percentage of states corresponds to rather high energies. In fact, for $x=0.44, g(E)$ ranges up to energies as large as $28 \mathrm{~K}$, and for $x=0.25$ the maximal range is only slightly reduced. 


\section{Results : specific heat and magnetization.}

From the density of states, in a noninteracting boson approximation, one can calculate both the specific heat $C_{\mathrm{H}}(T, B)$ and the magnetization per Eu atom, $M(T, B)$. For the large fields considered $M(0, B)$ should be given by the spin quantum number $s(=7 / 2)$, and the noninteractingboson approximation should be applicable as long as the relative deviation $\Delta M / M$, with $\Delta M:=M(0, B)-M(T, B)$, remains $\ll 1$. Then $\Delta M / M$ can be calculated from the integral

$$
(\Delta M / M)=\frac{1}{S} \int_{0}^{\infty} \frac{g(E) \mathrm{d} E}{\exp \left(E / k_{\mathrm{B}} T\right)-1},
$$

and the magnetic part $C_{\mathbf{H}}$ of the specific heat per Eu site by

$$
C_{\mathrm{H}} / k_{\mathrm{B}}=\frac{1}{\left(k_{\mathrm{B}} T\right)^{2}} \int_{0}^{\infty} \frac{g(E) E^{2} \exp \left(E / k_{\mathrm{B}} T\right) \mathrm{d} E}{\left[\exp \left(E / k_{\mathrm{B}} T\right)-1\right]^{2}} .
$$

In figure 2, results for $(\Delta M / M)$ are presented over $T$, for $B=6 \mathrm{~T}$ and $B=3 \mathrm{~T}$, and for $x=0.25$ and 0.54 , respectively [10]. From this figure it can be seen that $(\Delta M / M)$ remains smaller than 0.1 as long as $T \lesssim 5 \mathrm{~K}(10 \mathrm{~K})$ for $B=3 \mathrm{~T}(6 \mathrm{~T})$; this means that the non-interacting boson approximation should be applicable roughly up to these temperatures.

Figure 3 represents results for the specific heat. In figure $3 a$ the experimental results of [8], for $x=0.44$ and internal fields of $B=0,3.6$, and $6.6 \mathrm{~T}$, respectively, are compared with the theoretical predictions of the magnon theory. The theoretical results for $B=0$ are interpolated from former work of the author for $x=0.4$ and $x=0.5$, see [2]; they agree with experiment roughly

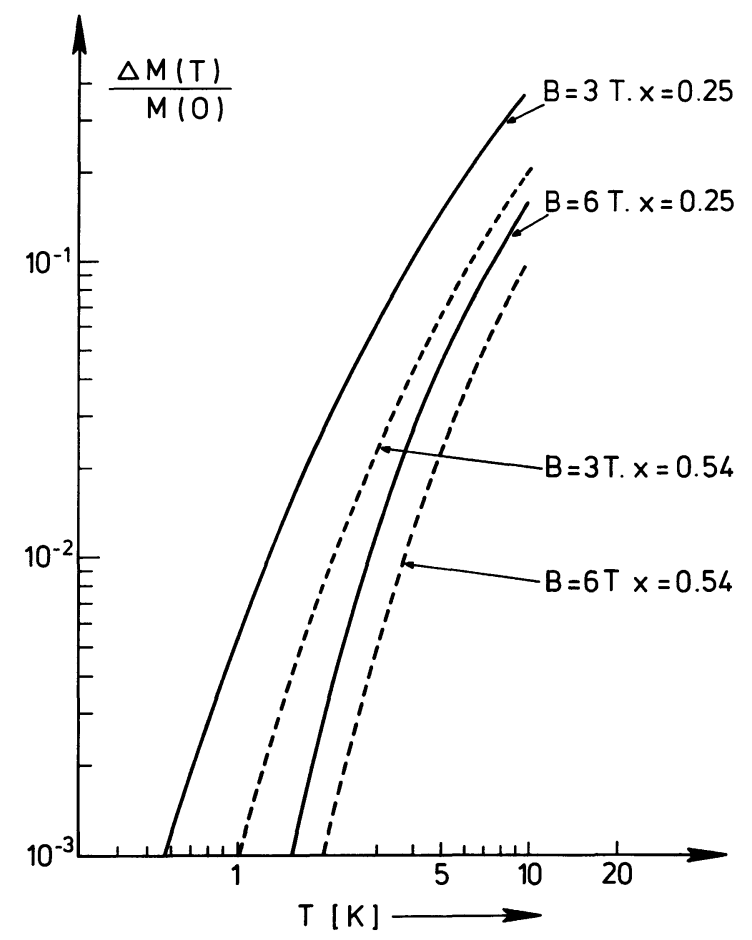

Fig. 2. - The relative decrease $\Delta M(T) / M(0)\{=1-[M(T) / M(0)]\}$ of the field-induced magnetization of $\mathrm{Eu}_{x} \mathrm{Sr}_{1-x} \mathrm{~S}$ spin glasses with $x=0.25$ and $x=0.54$ is presented over the temperature $T$ for $B=3$ and $B=6 \mathrm{~T}$. 


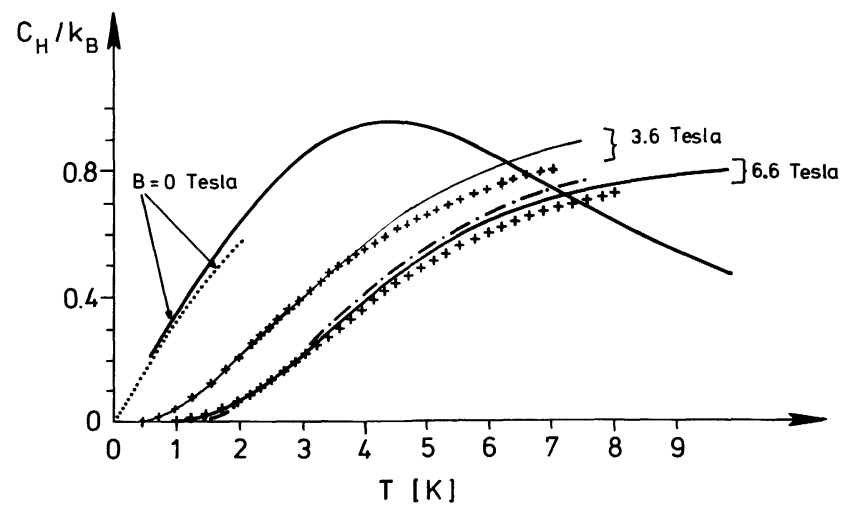

Fig. 3a. - For $\mathrm{Eu}_{x} \mathrm{Sr}_{1-x} \mathrm{~S}$-with $x=0.44$ experimental results of reference [8] for the specific heat per Eu atom at internal fields of $B=0,3.6$, and $6.6 \mathrm{~T}$ are presented over the temperature and compared with the theory. The solid lines are the experimental results, while the dotted curve is from the magnon theory of the author for $B=0$, taken from [2], whereas the crosses denote the result of the present calculation for $B=3.6$ and 6.6 T. Finally the dash-dotted curve, which looks alright for $T>2 \mathrm{~K}$, but deviates from the experiments below $2 \mathrm{~K}$, is the simple « Schottky fit 》 of reference [8], where instead of the collective magnon-like excitations incoherently superposed single spin excitations with an energy $E_{\left(B+B_{0}\right)}:=g \mu_{B}\left(B+B_{0}\right)$ are assumed, and $B_{0}=3.4 \mathrm{~T}$ is obtained as fit-parameter.
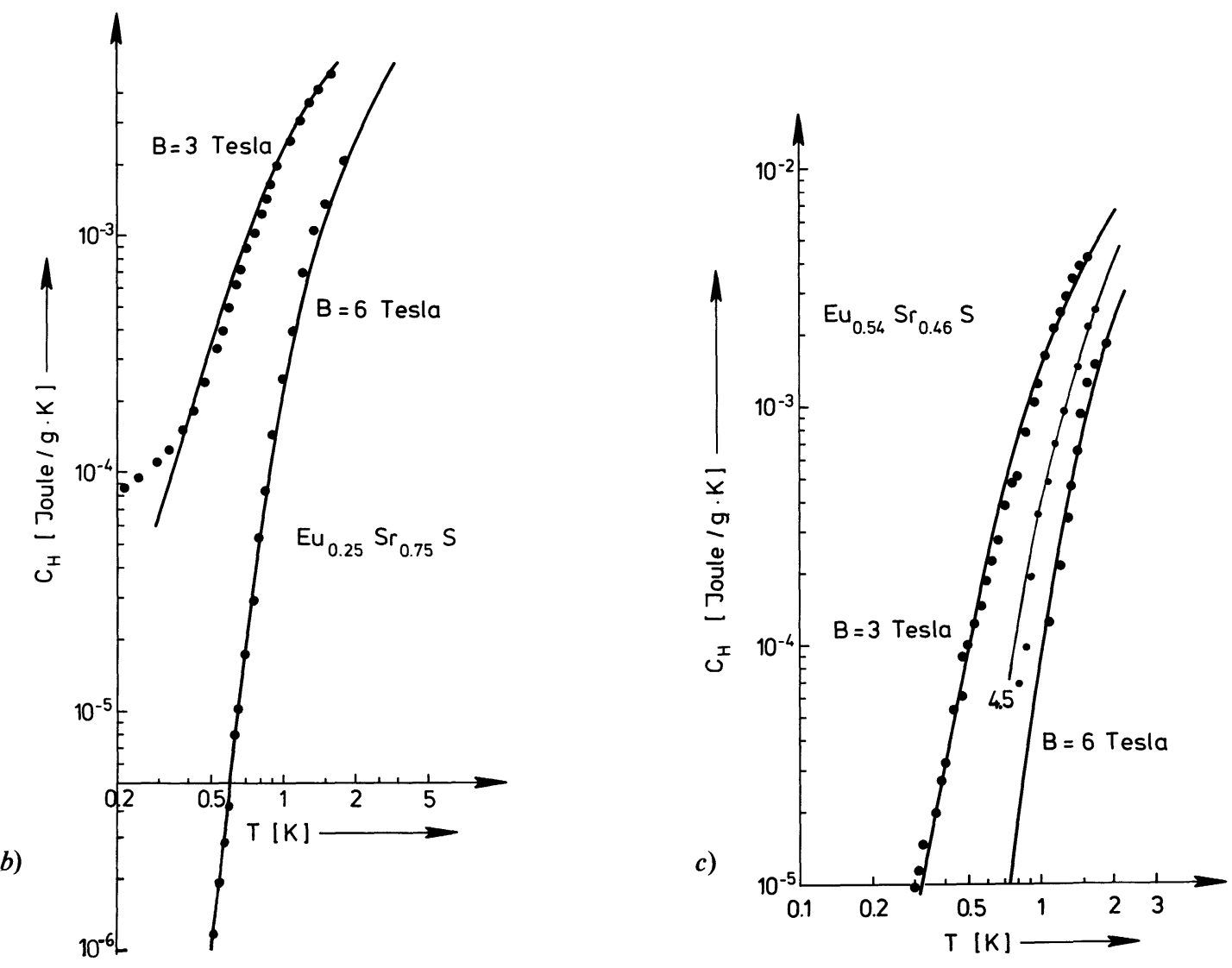

Fig. 3b, c. - The same as in figure 3a, however for $x=0.25$ and 0.54 for $B=3,4.5$, and $6 \mathrm{~T}$, below $2 \mathrm{~K}$. The solid lines are the numerical results of the present theory, while the black points represent the experimental results of reference [7]. 
up to $2 \mathrm{~K}$, whereas above that temperature, for $B=0$, the noninteracting-boson approximation would become unreasonable, leading to strong deviations between experiment and theory. However for $B=3.6$ and $6.6 \mathrm{~T}$ the situation is much more favourable, since in that case the theory is reasonable up to $5 \mathrm{~K}$ and $10 \mathrm{~K}$, respectively, see above. In fact, the theoretical results agree astonishingly well with the experiments both at high temperature and also at low temperatures. This should be contrasted with the "Schottky fit » of the authors of [8], i.e. the dash-dotted curve in figure 3 ; this fit would correspond to (incoherently superposed) single spin excitations at $E=E_{\left(B+B_{0}\right)}$, with $B_{0}=3.4 \mathrm{~T}$, see [8]. Although for $T \gtrsim 2 \mathrm{~K}$ the "Schottky fit " looks as convincing as the present magnon theory, it fails drastically for $T \lesssim 2 \mathrm{~K}$, in contrast to the magnon theory. This would become obvious, if for $T<2 \mathrm{~K}$ the results of [8] would be plotted logarithmically, i.e. in the way preferred by the authors of [7]. In fact, the excellence of the magnon theory can be seen most convincingly from figures $3 \mathrm{~b}$ and $3 \mathrm{c}$. There I compare my theoretical predictions for $x=0.25$ and $x=0.54$, respectively, with the very accurate experiments of [7]. The agreement is excellent, although the results cover a range of several orders of magnitude.

\section{Conclusions.}

Thus it has been shown that for $x=0.25,0.44$ and 0.54 the specific heat of $\mathrm{Eu}_{x} \mathrm{Sr}_{1-x} \mathrm{~S}$ spin glasses in internal fields of $3.6 \mathrm{~T}(6.6 \mathrm{~T})$, and for temperatures between $0.2 \mathrm{~K}$ and $5 \mathrm{~K}(10 \mathrm{~K})$, which is much larger than the spin glass freezing temperature for $B=0$, can be explained by a simple magnon theory, i.e. by starting from an aligned ground state and treating the magnon-like coherent superpositions of single spin excitations as non-interacting bosons. On the other hand, however, it can be shown from a calculation of the spectral function $S(\mathbf{q}, E)$, [11], that the thermal conductivity in the magnon channel should be extremely small, and that the drastic increase of the thermal conductivity with increasing $B$, which has been found in [8] and [12], and has already been interpreted by those authors in [12], comes from the phonon channel.

\section{References}

[1] Maletta, H., in Excitations in Disordered Systems, Ed. M. F. Thorpe (Plenum Press, New York) 1982, p. 431 ;

HUBER, D. L., ibid., p. 463.

[2] KReY, U., Z. Phys. B 38 (1980) 243.

[3] KREY, U., Z. Phys. B 42 (1981) 231.

[4] KREY, U., J. Magn. Magn. Mater. 28 (1982) 231.

[5] Meschede, D., Steglich, F., Felsch, W., Maletta, H. and Zinn, W., Phys. Rev. Lett. 44 (1980) 102.

[6] Ching, W. Y., Huber, D. L. and Leung, K. M., Phys. Rev. 21 (1980) 3708.

[7] von Löhneysen, H., van DEN Berg, R. and LeComte, G. V., Phys. Rev. B 31 (1985) 2920.

[8] Marcenat, C., Benoit, A., Briggs, A., Arzoumanian, C., De Goer, A. M., and Holtzberg, F., J. Physique Lett. 46 (1985) L-569.

[9] HAYDOCK, R., in [1], p. 29 and p. 43.

[10] Actually for $B=3 \mathrm{~T}$ and $x=0.25$ the gap should already be closed, since $3.3 \mathrm{~T}$ are needed to keep it open. However according to figure 1 , just above the gap (namely $0.3 \mathrm{~T}$, corresponding to $0.4 \mathrm{~K}$ ) the density of states is still extremely small ; therefore for $T>0.4 \mathrm{~K}$ one can simply erase this part of $g(E)$ and neglect the rearrangement of the ground state.

[11] KREY, U., unpublished.

[12] Arzoumanian, C., De Goer, A. M., Salce, B. and Holtzberg, F., J. Physique Lett. 44 (1983) L-39.

Commission paritaire $\mathbf{N}^{\circ} 59.024$

(C) Les Editions de Physique 1985

Directrice de la Publication : Jeanne BERGER

Imprimé en France. - JOUVE, 18, rue Saint-Denis, 75001 PARIS

$\mathrm{N}^{\circ} 34365$. Dépôt légal : Septembre 1985 